\title{
Assessment of Renal Function Status in Steady-State Sickle Cell Anaemic Children Using Urine Human Neutrophil Gelatinase-Associated Lipocalin and Albumin:Creatinine Ratio
}

\author{
Olatubosun Oladipupo Olawale ${ }^{a}$ Abiodun Folasade Adekanmbi ${ }^{b}$ \\ Ayobola Abimbola Sonugac Oyebola Oluwagbemiga Sonugad \\ Samuel Olufemi Akodu ${ }^{b}$ Morufat Mojisola Ogundeyi ${ }^{\mathrm{e}}$
}

aDepartment of Chemical Pathology and Immunology, Olabisi Onabanjo University Teaching Hospital, Sagamu, Nigeria; ${ }^{b}$ Department of Paediatrics, Olabisi Onabanjo University Teaching Hospital, Sagamu, Nigeria; 'Department of Biochemistry, Lead City University, Ibadan, Nigeria; ${ }^{d}$ Department of Chemical Pathology, University College Hospital,

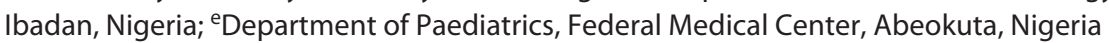

\section{Highlights of the Study}

- Renal function status of the children was assessed using urine albumin creatinine ratio and urine human neutrophil gelatinase-associated lipocalin.

- This study shows that kidney injury possibly begins early in childhood in sickle cell individuals and that albumin creatinine ratio is more sensitive than urine human neutrophil gelatinase-associated lipocalin in assessing renal injury in this age group.

- Findings of this study further emphasize the importance of periodic renal evaluations in sickle cell children, for early identification of those at risk of kidney injury.

\section{Keywords}

Sickle cell anaemia · Renal function · Urine albumin creatinine ratio $\cdot$ Human neutrophil gelatinase-associated lipocalin

\section{Abstract \\ Introduction: Sickle cell anaemia is characterized by defec- tive haemoglobin synthesis and is associated with both en- docrine and metabolic alterations. The effects of this clinical condition on kidney function are multifactorial and often be- gin early in childhood. This study aims to assess renal func- tion in children with sickle cell anaemia using urine}

albumin:creatinine ratio (ACR) and urine human neutrophil gelatinase-associated lipocalin (NGAL). Methods: This casecontrol study was conducted on 200 children aged 5-15 years in 2 tertiary hospitals in South West Nigeria: 150 were of haemoglobin $\mathrm{S}$ genotype and 50 were of haemoglobin $A$ genotype. Serum urea, creatinine, urine albumin, and NGAL were assayed by known standard methods. eGFR, urine ACR, and urine NGAL/creatinine ratio (urine NCR) were calculated. Results: The weight, height, BMI, systolic blood pressure, plasma urea, plasma creatinine, and spot urine creatinine of the HbS genotype children were significantly lower compared to that of the HbA genotype children. The eGFR, spot urine albumin, and urine ACR were significantly higher in the karger@karger.com www.karger.com/mpp

Karger $\stackrel{\text { ' }}{5}$ GOPEN ACCESS
(C) 2021 The Author(s)

Published by S. Karger AG, Basel

This is an Open Access article licensed under the Creative Commons Attribution-NonCommercial-4.0 International License (CC BY-NC) (http://www.karger.com/Services/OpenAccessLicense), applicable to the online version of the article only. Usage and distribution for commercial purposes requires written permission.
Correspondence to:

Oyebola Oluwagbemiga Sonuga, oyebolasonuga@yahoo.com 
$\mathrm{HbS}$ group compared to the $\mathrm{HbA}$ group. There was no significant difference in the spot urine NGAL and urine NCR between the 2 groups, though both were higher in the $\mathrm{HbS}$ group compared to the HbA group. Conclusions: Kidney injury probably starts early in childhood in sickle cell individuals as indicated by the higher urine ACR detected in them. We infer that urine NGAL and $U N C R$ are not sensitive markers of kidney disease especially in young sickle cell individuals possibly because of the hyperfiltration present at this age.

(c) 2021 The Author(s)

Published by S. Karger AG, Basel

\section{Introduction}

Sickle cell disease (SCD) is an autosomal recessive disorder resulting from mutation in the $\beta$-globin gene of haemoglobin, leading to production of an unstable isoform, haemoglobin $\mathrm{S}(\mathrm{HbS}$ ) which when deoxygenated can polymerize causing sickling of the red blood cell, vaso-occlusion, and haemolysis. SCD includes sickle cell anaemia (SCA) which is due to homozygous inheritance of haemoglobin $\mathrm{S}(\mathrm{HbS})$, consequently the most severe form of SCD. The prevalence of the sickle cell trait in many tropical African countries including Nigeria ranges between 20 and $30 \%$ of the population [1].

Sickle cell nephropathy is a common and serious complication of SCA that begins in childhood and may progress to overt renal failure. Structural and functional changes may occur in the kidney as a result of the haemodynamic changes associated with chronic anaemia and renal hypoxia resulting from the recurrent vaso-occlusion and haemolysis-related endothelial dysfunction. These changes present as concentrating defect, renal insufficiency, haematuria, proteinuria, and hypertension which eventually progress to CKD [2].

Chronic renal failure has been increasingly diagnosed in $\mathrm{HbS}$ patients, and in some countries, it is one of the most common causes of death among HbS patients over 40 years of age. The prevalence of renal failure in SCD ranges from 5 to $18 \%$ [3]. Studies have shown that preventive strategies can significantly reduce the burden of renal diseases $[4,5]$ hence the importance of detecting kidney injury at early age especially in children with the haemoglobin $S$ genotype.

There are several available serum and urine biomarkers of renal injury including urea, creatinine, urine albumin, and urine human neutrophil gelatinase-associated lipocalin (NGAL), but none of them could accurately predict the outcome of the disease at the initial stages. Kidney diseases can be diagnosed on the basis of serum creatinine concentration and estimated glomerular filtration rate (eGFR), albuminuria, renal imaging, and histology following renal biopsy [6].

The plasma urea and creatinine are the commonest and generally recognized parameters utilized in the assessment of renal functions, such that their elevated concentration is a pointer to impaired renal function. A number of extra-renal factors affect the plasma concentrations of urea and creatinine, so limiting their significance as a test of kidney injury and therefore should not be used alone [7]. Plasma creatinine is used in the estimation of GFR which is the optimal way to assess kidney function and in conjunction with albuminuria can help determine the extent of CKD in an individual.

GFR is measured using plasma or urinary clearance of an exogenous filtration marker such as inulin; however, it is a complex procedure and generally not routinely performed; therefore, GFR is usually estimated from an individual's plasma creatinine concentration in combination with demographic factors such as age, race, and gender using various formulae [8]. A decrease in GFR precedes onset of kidney failure such that persistently reduced GFR is a specific diagnostic criterion for CKD. One of the major manifestations of renal injury observed in children with SCA is glomerular impairment characterized by an early increase in GFR associated with high or overt albuminuria, followed by a gradual decline in GFR and finally CKD [9].

Albuminuria, an increase in urinary excretion of albu$\mathrm{min}$, is also a sensitive marker of glomerular damage that may indicate early CKD, and it is considered to be a relevant biomarker of early glomerular damage in patients with SCD. A routine dipstick is not sensitive enough to detect small amounts of urinary protein (albumin), and therefore to evaluate albuminuria, it is recommended to measure urine albumin-to-creatinine ratio (uACR) in a spot urine sample because urine albumin varies greatly throughout the day while creatinine is excreted in a relatively steady rate. Although the 24-h urine collection is the "gold standard," to quantify urinary protein, ACR in the spot urine specimen correlates well with 24-h urine collections, and it is less cumbersome. It has been shown that in sickle cell individuals, a prolonged period of highalbuminuria precedes gross persistent proteinuria, followed by renal failure as age increases [10].

Rapid rise in levels of urine NGAL compared to levels of serum creatinine in response to AKI is one of its benefits over creatinine, and contrary to these conventional markers, NGAL is not considered a marker of renal function but a reflection of structural damage of renal cells. 
NGAL therefore shows a progression of the early renal structural damage occurring during kidney disease despite normal GFR [11]. It is proposed that UNGAL estimation should be done along with urine creatinine measurements and uNGAL/creatinine ratio (uNCR) calculated, and this is due to high biological variability of urinary NGAL (uNGAL), especially in CKD [12]. It has been reported that there is a positive association between uNGAL, albuminuria, and kidney disease progression and a negative correlation between UNGAL, UNCR, and eGFR, irrespective of the level of albuminuria [13]. Additionally, uNGAL seems to be a better biomarker in children and adolescents as its assessment is less invasive than assessing even serum cystatin $\mathrm{C}$ level and that it can be measured using a single urine sample [14]. The interpretation of the result, however, is often difficult in paediatric population because of lack of age- and sex-specific normative values. Previous published urine NGAL values ranged from $1.64(0.25-5.77) \mathrm{ng} / \mathrm{mL}$ in healthy children [15] to $5(2-150) \mathrm{ng} / \mathrm{mL}$ in very low birth weight infants [16].

There are only few publications regarding kidney injury in paediatric patients with SCD, and information on the renal status of children with the haemoglobin S genotype in Nigeria is sparse and conflicting, thus the need for this present study. Early detection of the HbS genotype children who are at a high risk of kidney impairment is highly significant as it helps to apply measures which can delay progressive kidney dysfunction This present study therefore compared the renal function using eGFR, urine ACR, urine NGAL, and urine NCR in HbS and apparently healthy $\mathrm{HbA}$ children, as a means to assess the effect of SCA on the renal indices at early childhood.

\section{Methods}

\section{Study Participants}

This is a case-control study carried out among 200 children aged between 5 and 15 years in 2 tertiary hospitals from South West, Nigeria, over a period of 6 months (April-November 2019). One hundred fifty children were of HbS genotype and 50 were of $\mathrm{HbA}$ genotype. The study participants were selected from children attending the paediatric haematology clinic after satisfying the inclusion criteria using a nonrandom sampling technique. The study was approved by the Olabisi Onabanjo University Teaching Hospital Health Research and Ethics Committee (OOUTH/ HREC/257/2019).

\section{Data Collection}

A questionnaire-based interview was used to collect information on demographic characteristics and clinical measurements. Informed written consent was obtained from all participants' guard-
Table 1. Baseline characteristics of children with HbS genotype and children with $\mathrm{HbA}$ genotype

\begin{tabular}{llcc}
\hline Parameters & $\begin{array}{l}\text { HbS (test), } \\
(n=150)\end{array}$ & $\begin{array}{l}\text { HbA (control), } \\
(n=50)\end{array}$ & $p$ value \\
\hline Age, years & $9.14 \pm 0.26$ & $10.9 \pm 0.44$ & 0.21 \\
Weight, kg & $26.5 \pm 0.71$ & $36.5 \pm 1.91$ & $<0.001^{*}$ \\
Height, m & $1.29 \pm 0.01$ & $1.42 \pm 0.02$ & $0.003^{*}$ \\
BMI, kg/m ${ }^{2}$ & $15.3 \pm 0.16$ & $17.6 \pm 0.58$ & $<0.001^{*}$ \\
SBP, mm Hg & $90.7 \pm 1.12$ & $105.3 \pm 0.03$ & $0.041^{*}$ \\
DBP, mm Hg & $51.8 \pm 0.95$ & $63.1 \pm 1.71$ & 0.19 \\
\hline
\end{tabular}

There was no significant difference in the age and DBP of children with sickle cell anaemia when compared with controls. The weight, height, BMI, and SBP were significantly lower in the HbS group when compared with the HbA group. BMI, body mass index; SBP, systolic blood pressure; DBP, diastolic blood pressure. $* p<0.05$.

ians after educating them on the benefits and relevance of the study. Clinical measurements which included weight $(\mathrm{kg})$ and height $(\mathrm{m})$ were measured with participants in light clothing without shoes using a stadiometer and the stadiometer's head piece, respectively. BMI was calculated as weight $/$ height $^{2}\left(\mathrm{~kg} / \mathrm{m}^{2}\right)$. Blood pressure was measured twice (10 min apart), on the left arm of participants in a relaxed, sitting position with the arm supported at heart level, using a standard mercury sphygmomanometer. The mean of the 2 readings was calculated to obtain the final blood pressure.

Five millilitres of venous blood was withdrawn from each participant and dispensed into a lithium heparin sample bottle for plasma urea and creatinine estimation, while $10 \mathrm{~mL}$ of the spot urine specimen was collected for urine albumin, urine creatinine, and NGAL estimation. The lithium heparin specimen bottles were centrifuged at 3,000 $\mathrm{g}$ for $15 \mathrm{~min}$ using the Uniscope Laboratory centrifuge, model SM 112 (Surgifriend Medicals, England), and plasma decanted into respective well-labelled plain bottles. Respective plasma and urine samples were stored at $-20^{\circ} \mathrm{C}$ using the freezer compartment of the SCANFROST model SFVFFF 350 until analysis within a period of 3 months.

\section{Assay Methods}

Serum urea was measured by the urease-Berthelot colourimetric method, serum and urine creatinine was quantified using the alkaline picrate colourimetric method, and urine albumin was quantified using immunoturbidimetric assay while urine NGAL was measured by the ELISA method. eGFR was calculated using the revised bedside Schwartz formula. uACR and uNCR ratios were also calculated.

\section{Statistical Analysis}

Statistical analysis was done using the IBM statistical package for social sciences (SPSS) version 23.0. The data are expressed as mean \pm standard error. The Student's $t$ test was used for comparisons of means while Pearson's correlation was done to find associations between eGFR and plasma urea, creatinine, urine ACR, urine NGAL, and urine NGAL:creatinine ratio. $p$ values $<0.05$ were considered significant. 


\section{Discussion}

In this present study, the homozygous SCD children were of shorter stature when compared with their agematched control (HbA genotype children) as shown in "Table 1," and this is similar to reports by Nogueira et al. [17] and Al-Saqladi et al. [18]. Thus, in agreement with the previous studies, this present study shows that sickle cell anaemic children are growth deficient compared to children with $\mathrm{HbA}$.

We observed that children with $\mathrm{HbS}$ have lower plasma urea and plasma creatinine compared to HbA controls as shown in "Table 2"; this is similar to previous reports by Aloni and Nkee [19] and Aleem [20]. Also, in agreement with previous reports submitted by Aleem [20] and Aygun et al. [21] that elevated GFR is predominant in sickle cell paediatric series, the eGFR in this present study is higher in $\mathrm{HbS}$ children compared to $\mathrm{HbA}$ children "as shown in Table 2," though both are in the normal GFR category for CKD staging. This elevated eGFR referred to as hyperfiltration is common in young patients suffering from SCD due to glomerular hypertrophy such that in poor-resource settings, hyperfiltration is a major indicator of deterioration in renal function, which occurs earlier than decreased creatinine clearance and/or proteinuria [22]. The amount of creatinine produced in the body each day is relatively constant, and it is related to the muscle mass; this coupled with the glomerular hyperfiltration might explain the lower concentrations of creatinine and urea in $\mathrm{HbS}$ who are of smaller stature compared to their $\mathrm{HbA}$ counterpart.

This study showed that urine albumin and urine albumin creatinine ratio of the sickle cell children are significantly higher than those of the HbA counterpart "as shown in Table 2." This is similar to previous findings by Ranque et al. [23]. There is also moderately increased albuminuria in the sickle cell anaemic participants indicating the stage $\mathrm{A} 2 \mathrm{CKD}$ albuminuria category, while the $\mathrm{HbA}$ participants are in the normal albuminuria category. It is known that albuminuria is a sensitive marker of glomerular damage; therefore, it can be inferred from this study that the sickle cell anaemic children may be in the early phase of kidney damage with compensatory increase in glomerular filtration which may be responsible for the enhanced glomerular passage of albumin.

It is imperative to note that serum creatinine and its derived eGFR might be of limited diagnostic value in detecting early renal dysfunction due to many factors that affect the generation and excretion of creatinine. It has also been reported that not all individuals with conditions
Table 2. Renal indices of HbS genotype and HbA genotype children

\begin{tabular}{lccc}
\hline Parameters & $\begin{array}{l}\text { HbS (test) } \\
(n=150)\end{array}$ & $\begin{array}{l}\text { HbA (control) } \\
(n=50)\end{array}$ & $p$ value \\
\hline Plasma urea, mg/dL & $16.8 \pm 1.30$ & $23.1 \pm 1.89$ & $<0.001^{*}$ \\
Plasma Cr, mg/dL & $0.54 \pm 0.01$ & $0.75 \pm 0.05$ & $<0.001^{*}$ \\
Spot urine Cr, mmol/L & $5.47 \pm 0.34$ & $10.2 \pm 1.41$ & $<0.001^{*}$ \\
eGFR, mL/min/1.73 m ${ }^{2}$ & $122.5 \pm 9.0$ & $104.7 \pm 3.8$ & $0.02^{*}$ \\
Spot urine albumin, mg/L & $22.8 \pm 1.8$ & $9.73 \pm 0.68$ & $0.002^{*}$ \\
Urine ACR, mg/mmol & $7.37 \pm 1.09$ & $1.75 \pm 0.25$ & $<0.001^{*}$ \\
Spot urine NGAL, mg/L & $0.24 \pm 0.01$ & $0.20 \pm 0.01$ & 0.89 \\
Urine NCR, mg/mmol & $0.06 \pm 0.01$ & $0.04 \pm 0.01$ & 0.19 \\
\hline
\end{tabular}

The plasma urea, plasma creatinine, and spot urine creatinine of HbS children are significantly lower compared to HbA children. The eGFR, spot urine albumin, and urine albumin creatinine ratio were significantly higher in the $\mathrm{HbS}$ group compared to the $\mathrm{HbA}$ group. There was no significant difference in the spot urine NGAL and urine NGAL creatinine ratio (urine NCR) when compared in the 2 groups, though both were higher in the $\mathrm{HbS}$ group compared to the $\mathrm{HbA}$ group. Urine ACR, urine albumin:creatinine ratio; urine NGAL, urine human neutrophil gelatinase-associated lipocalin; urine NCR, urine human neutrophil gelatinase-associated lipocalin:creatinine ratio. ${ }^{*} p<0.05$.

such as diabetic nephropathy have increased albuminuria, and therefore a search for new markers for kidney damage seems necessary.

NGAL and kidney injury molecule-1 are released by injured tubular epithelium cells, and interleukin-18 is an inflammatory mediator released during AKI. These molecules, which take part in the early pathophysiological changes in AKI, have been recently evaluated as potential new biomarkers. Tissue inhibitor of metalloproteinase- 2 and insulin-like growth factor binding protein- 7 both act to block the G1 stage of the renal tubular epithelial cell cycle during AKI [24]. With varying degrees of accuracy, these markers all provide information about the state of the kidney much earlier than do changes in function (i.e., serum creatinine).

NGAL, a member of the lipocalin protein family, has been identified as one of the most useful biomarkers to detect kidney disease in its early stages. Available reports focus mainly on the role of NGAL as a biomarker of AKI, but it is worthy of note that it may also serve as a marker of CKD, especially diabetic nephropathy [25].

There is a paucity of reports on urine NGAL in sickle cell children especially in Nigeria, and this present study might be among the very few to investigate renal status in these group of children using urine NGAL. In this present study, the urine NGAL and urine NGAL:creatinine ratio are high- 
er in $\mathrm{HbS}$ participants compared to HbA controls "as shown in Table 2," though not significantly different; this is similar to the findings of Woo et al. [26] and Atere et al. [11]. Atere et al. [11] looked at NGAL as a marker for AKI among sickle cell subjects (both steady state and vaso-occlusive crisis state) aged 18-60 years and reported significantly higher NGAL in them compared to controls while Woo et al. [26] reported significantly higher urinary NGAL levels in diabetic CKD patients than in healthy controls. The participants of this present study are children, aged 5-15 years with no AKI or CKD; this might explain the lack of significant difference in the measured urine NGAL or calculated urine NCR as compared to studies by Woo et al. [26] and Atere et al. [11] where adult patients with known AKI or CKD are compared with apparently healthy controls. Research has shown that in patients with CKD, elevated urine NGAL is a good predictor of CKD progression [27] and urine NGAL been a marker of structural damage of renal cells, then it can be proposed from this present study that some level of structural kidney injury though insignificant might be present in sickle cell individuals from childhood which might progress to AKI and CKD as they grow older.

The current study shows a strong negative correlation between the eGFR and the plasma creatinine of sickle cell children as shown in "Table 3," and this is similar to the finding of Srivastava et al. [28] in which it was reported that serum creatinine increases in renal failure correlating with a decrease in GFR forming a curvilinear relationship. These findings efficiently demonstrated that decline in glomerular filtration is associated with rise in plasma creatinine concentrations.

We did not observe any correlation between the eGFR, measured urine NGAL, and calculated urine NCR shown in "Table 3" as compared to the findings of Woo et al. [26] and Atere et al. [11] in which urinary NGAL level showed a significant inverse correlation with eGFR in the studied participants. Atere et al. [11] reported that NGAL correlates positively with the duration of SCD mainly when eGFR is decreased, and this might also explain the lack of correlation in this present study where participants are children with hyperfiltration; therefore, NGAL might not be a sensitive marker of kidney disease in sickle cell children probably in the absence of AKI or CKD.

\section{Conclusions}

Kidney injury possibly begins early in childhood in sickle cell individuals as indicated by the moderately but significantly increased albuminuria found in them. It is
Table 3. Correlation of some parameters with eGFR in the HbS group

\begin{tabular}{lcc}
\hline Correlating pair & $R$ & $p$ value \\
\hline eGFR versus plasma urea & -0.064 & 0.435 \\
eGFR versus plasma creatinine & -0.790 & $<0.001^{*}$ \\
eGFR versus urine ACR & 0.137 & 0.067 \\
eGFR versus urine NGAL & -0.258 & 0.154 \\
eGFR versus urine NCR & -0.132 & 0.470 \\
\hline
\end{tabular}

There is a strong negative correlation between eGFR and plasma creatinine $(r=-0.790, p=<0.001)$. Urine ACR, urine albumin:creatinine ratio; urine NGAL, urine human neutrophil gelatinase-associated lipocalin; urine NCR, urine human neutrophil gelatinase-associated lipocalin:creatinine ratio. ${ }^{*} p<0.05$.

likely that urine NGAL and UNCR might not be a sensitive marker of kidney disease in sickle cell individuals especially when they are young possibly because of the hyperfiltration present at this phase of their life. Limitations of this study include its small sample size and the use of a single marker of structural kidney injury. However, findings of this study buttressed the known fact that it is crucial to perform periodic renal evaluations in sickle cell children, so as to detect early those at risk of kidney injury and apply measures which can delay progressive kidney impairment.

\section{Acknowledgments}

We are thankful to the study participants for making this study possible. We also acknowledge the help of the entire Department of Paediatric Haematology unit of both Olabisi Onabanjo University Teaching Hospital and Federal Medical centre, Abeokuta, for their assistance.

\section{Statement of Ethics}

All procedures performed in this study involving human participants were in accordance with the ethical standards of the Olabisi Onabanjo University Teaching Hospital Health Research and Ethics Committee "Reference No. OOUTH/HREC/257/2019" and with the 1964 Helsinki Declaration and its later amendments or comparable ethical standards.

\section{Conflict of Interest Statement}

The authors declare no conflicts of interest. 


\section{Funding Sources}

No financial support was obtained.

\section{Author Contributions}

Conception and design for the current study, O.O.O.; recruitment of study participants and sample collection, A.F.A., S.O.A., M.M.O.; statistical analysis, A.A.S.; collation of data and writing up of manuscript; O.O.S.; final approval of the version to be published all the authors.

\section{Data Availability Statement}

All data generated or analyzed during this study are included in this paper. Further enquiries can be directed to the corresponding author, O.O.S. The data that support the findings of this study are not publicly available because the information contained in it can compromise the privacy of the research participants; but they are available from the corresponding author, O.O.S., upon reasonable request.

\section{References}

1 David AN, Jinadu MY, Wapmuk AE, Gbajabiamila TA, Okwuzu JO, Herbertson EC, et al. Prevalence and impact of sickle cell trait on the clinical and laboratory parameters of HIV infected children in Lagos, Nigeria. Pan Afr Med J. 2018 Oct 15;31:113.

2 Day TG, Drasar ER, Fulford T, Sharpe CC, Thein SL. Association between hemolysis and albuminuria in adults with sickle cell anemia. Haematologica. 2012;97(2):201-5.

3 Saborio P, Scheinman JI. Sickle cell nephropathy. J Am Soc Nephrol. 1999 Jan;10(1):187-92.

4 Du Y, Zappitelli M, Mian A, Bennett M, Ma Q, Devarajan P, et al. Urinary biomarkers to detect acute kidney injury in the pediatric emergency center. Pediatr Nephrol. 2011 Feb; 26(2):267-74

5 Parikh CR, Devarajan P, Zappitelli M, Sint K, Thiessen-Philbrook H, Li S, et al. Postoperative biomarkers predict acute kidney injury and poor outcomes after pediatric cardiac surgery. J Am Soc Nephrol. 2011;22(9):1737-47.

6 Żyłka A, Gala-Błądzińska A, Dumnicka P, Ceranowicz P, Kuźniewski M, Gil K, et al. Is urinary NGAL determination useful for monitoring kidney function and assessment of cardiovascular disease? A 12-month observation of patients with type 2 diabetes. Dis Markers. 2016;2016:8489543.

7 Edmund L, David JL, Christopher PP, editors. Chapter 24 kidney function. Tietz textbook of clinical chemistry and molecular diagnostics. 4th ed. USA: Elsevier Saunders; 2006. p. 797835.

8 National Kidney Foundation, 2011.

9 Nath KA, Hebbel RP. Sickle cell disease: renal manifestations and mechanisms. Nat Rev Nephrol. 2015;11(3):161-71.

10 Eke CB, Okafor HU, Ibe BC. Prevalence and correlates of microalbuminuria in children with sickle cell anaemia: experience in a Tertiary Health Facility in Enugu, Nigeria. Int J Nephrol. 2012;2012:240173. Epub 2012 Sep 28.

11 Atere AD, Ajani OF, Akinbo DB, Adeosun OA, Anombem OM. Serum levels of neutrophil gelatinase-associated lipocalin (NGAL) as predictor of acute kidney injury in sickle cell subjects. J Biomed Sci. 2018;7(4):17.
12 Helmersson-Karlqvist J, Arnlöv J, Larsson A. Day-to-day variation of urinary NGAL and rational for creatinine correction. Clin Biochem. 2013 Jan;46(1-2):70-2.

13 Wu J, Ding Y, Zhu C, Shao X, Xie X, Lu K, et al. Urinary TNF- $\alpha$ and NGAL are correlated with the progression of nephropathy in patients with type 2 diabetes. Exp Ther Med. 2013;6(6):1482-8.

14 Ataei N, Ameli S, Yousefifard M, Oraei A, Ataei F, Bazargani B, et al. Urinary neutrophil gelatinase-associated lipocalin (NGAL) and cystatin $\mathrm{C}$ in early detection of pediatric acute kidney injury; a Diagnostic Accuracy Study. Emerg. 2018;6(1):e2.

15 Wasilewska A, Taranta-Janusz K, Dębek W, Zoch-Zwierz W, Kuroczycka-Saniutycz E. KIM-1 and NGAL: new markers of obstructive nephropathy. Pediatr Nephrol. 2011 Apr; 26(4):579-86.

16 Huynh TK, Bateman DA, Parravicini E, Lorenz JM, Nemerofsky SL, Sise ME, et al. Reference values of urinary neutrophil gelatinaseassociated lipocalin in very low birth weight infants. Pediatr Res. 2009;66(5):528-32.

17 Nogueira ZD, Boa-Sorte N, Leite MEQ, Kiya MM, Amorim T, da Fonseca SF. Breastfeeding and the anthropometric profile of children with sickle cell anemia receiving followup in a newborn screening reference service. Rev Paul Pediatr. 2015;33(2):154-9.

18 Al-Saqladi A-WM, Cipolotti R, Fijnvandraat $\mathrm{K}$, Brabin BJ. Growth and nutritional status of children with homozygous sickle cell disease. Ann Trop Paediatr. 2008;28(3):165-89.

19 Aloni MN, Nkee L. Challenge of managing sickle cell disease in a pediatric population living in kinshasa, democratic republic of congo: a sickle cell center experience. Hemoglobin. 2014;38(3):196-200.

20 Aleem A. Renal abnormalities in patients with sickle cell disease: a single center report from Saudi Arabia. Saudi J Kidney Dis Transpl. 2008;19:194-9.
21 Aygun B, Mortier NA, Smeltzer MP, Hankins JS, Ware RE. Glomerular hyperfiltration and albuminuria in children with sickle cell anemia. Pediatr Nephrol. 2011;26(8):1285-90.

22 Aloni MN, Ngiyulu RM, Gini-Ehungu JL, Nsibu CN, Ekila MB, Lepira FB, et al. Renal function in children suffering from sickle cell disease: challenge of early detection in highly resource-scarce settings. PLoS One. 2014; 9(5):e96561.

23 Ranque B, Menet A, Diop IB, Thiam MM, Diallo D, Diop S, et al. Early renal damage in patients with sickle cell disease in sub-Saharan Africa: a multinational, prospective, cross-sectional study. Lancet Haematol. 2014 Nov;1(2):e64-73. Epub 2014 Oct 28.

24 Kashani K, Al-Khafaji A, Ardiles T, Artigas A, Bagshaw SM, Bell M, et al. Discovery and validation of cell cycle arrest biomarkers in human acute kidney injury. Crit Care. 2013; 17(1):R25.

25 Siew ED, Ware LB, Gebretsadik T, Shintani A, Moons KG, Wickersham N, et al. Urine neutrophil gelatinase-associated lipocalin moderately predicts acute kidney injury in critically ill adults. J Am Soc Nephrol. 2009;20(8): 1823-32.

26 Woo KS, Choi JL, Kim BR, Kim JE, An WS, Han JY. Urinary neutrophil gelatinase-associated lipocalin levels in comparison with glomerular filtration rate for evaluation of renal function in patients with diabetic chronic kidney disease. Diabetes Metab J. 2012;36(4): 307-13.

27 Bolignano D, Lacquaniti A, Coppolino G, Donato V, Campo S, Fazio MR, et al. Neutrophil gelatinase-associated lipocalin (NGAL) and progression of chronic kidney disease. Clin J Am Soc Nephrol. 2009;4(2):337-44.

28 Srivastava M, Gaikwad RV, Samad A, Sharma B, Srivastava A. Relationship of Serum Creatinine and Glomerular Filtration Rate by 99mTc-DTPA Scintigraphy in Dogs with Renal Failure. Asian J Anim Sci. 2011;5:381386. 